\title{
Long wavelength optical coherence tomography for painted objects
}

\author{
C. S. Cheung ${ }^{1}$, M. Tokurakawa ${ }^{2}$, J. M. O. Daniel ${ }^{2}$, W. A. Clarkson ${ }^{2}$, H. Liang $^{1 *}$ \\ ${ }^{1}$ School of Science \& Technology, Nottingham Trent University, Nottingham NG1 1 8BS, UK \\ ${ }^{2}$ Optoelectronics Research Centre, University of Southampton, Highfield, SO17 1BJ, UK. \\ haida.liang@ntu.ac.uk
}

\begin{abstract}
Optical Coherence Tomography has been successfully applied to the imaging of painted objects in recent years. However, a significant limitation is the low penetration depth of OCT in paint because of the high opacity of paint due to either scattering or absorption. It has been shown that the optimum spectral window for OCT imaging of paint layers is around $2.2 \mu \mathrm{m}$ in wavelength. In this paper, we demonstrate a $1950 \mathrm{~nm}$ OCT for imaging painted objects using a superfluorescent fiber source at low power.
\end{abstract}

Keywords: Optical Coherence Tomography, Fourier domain, ultra-high resolution, micron-scale

\section{INTRODUCTION}

Optical Coherence Tomography (OCT) is a non-invasive, non-contact imaging technique originally designed for in vivo imaging of the eye and other biological tissues. OCT is a fast scanning Michelson interferometer capable of 3D imaging of subsurface microstructure. In recent years, OCT has been successfully applied to the non-invasive imaging of paintings and other cultural artifacts. Apart from the non-invasive examination of the stratigraphy of paint and varnish layers $[1,2]$, OCT has also been shown to be the most sensitive technique for revealing preparatory underdrawings beneath paint layers owing to its high dynamic range and depth selection capabilities [2,3]. OCT has the potential to become a routine non-invasive tool in museums allowing cross-section imaging anywhere on an intact object where there are no other methods of obtaining subsurface information. While current OCTs have shown potential in this field, they are optimized for biomedical applications. One major limitation of OCT imaging is the probing depth through highly scattering paint. OCT systems for biomedical applications are generally restricted to wavelengths between $800 \mathrm{~nm}$ and $1300 \mathrm{~nm}$ for the best compromise between water absorption and tissue scattering. However, the requirements of art conservation are very different since most paints are highly scattering and the transparency is not limited by water absorption as in the case of biological tissue. A recent survey of the transparency of historical artists' pigments over the spectral range of $400 \mathrm{~nm}-2400 \mathrm{~nm}$ has shown that the optimum spectral window for OCT imaging of paint layers is in a spectral window around $2.2 \mu \mathrm{m}$ [4]. Figure 1 shows that median spectral transparency of a sample of common pre-1 $9^{\text {th }}$ century artists' paint in two common binding media, egg tempera and linseed oil. Excluding the lake pigments (which are all highly transparent at wavelength $>600 \mathrm{~nm}$ ) in the sample of 45 pigments studied, over $30 \%$ of the paint samples are $>5$ times more transparent at $2.2 \mu \mathrm{m}$ than at $800 \mathrm{~nm}$, and $\sim 25 \%$ of the paint samples are $>2$ times more transparent at $2.2 \mu \mathrm{m}$ than at $1.5 \mu \mathrm{m}$. Off-the-shelf OCT sources are commonly found around $800 \mathrm{~nm}, 1000 \mathrm{~nm}, 1300 \mathrm{~nm}$ and $1500 \mathrm{~nm}$ and few OCT systems have been built beyond $1300 \mathrm{~nm}$. Therefore, OCT at long wavelength $(\sim 2 \mu \mathrm{m})$ will give the largest probing depth and push OCT imaging towards matching the information content given by the invasive method currently employed in museums of microscopic examination of sample cross-sections.

As a proof of concept, we demonstrate in this paper a time-domain OCT using a broadband Tm-doped superfluorescent fiber source generated through the process of amplified spontaneous emission (ASE) [5]. One of the attractive characteristics of such sources is the increased bandwidth with decreasing power. Low power is a requirement of noninvasive imaging of cultural heritage.

\footnotetext{
Optics for Arts, Architecture, and Archaeology IV, edited by Luca Pezzati,

Piotr Targowski, Proc. of SPIE Vol. 8790, 87900 - (c) 2013 SPIE

CCC code: $0277-786 \mathrm{X} / 13 / \$ 18 \cdot$ doi: $10.1117 / 12.2021700$
} 


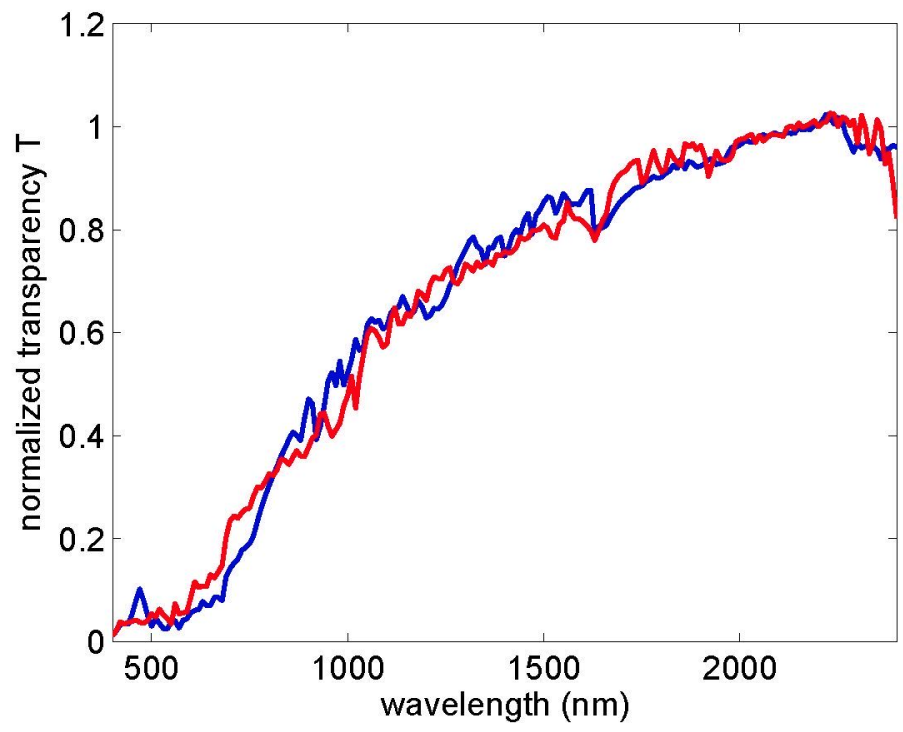

Figure 1. Optimum spectral window for pigment transparency: median spectral transparency normalized at $2.2 \mu \mathrm{m}$ for pigments in use before the nineteenth century but excluding the lake pigments which are transparent at all wavelengths. Those in an oil medium are shown in blue and those in egg tempera are shown in red [4].

\section{INSTRUMENT SETUP}

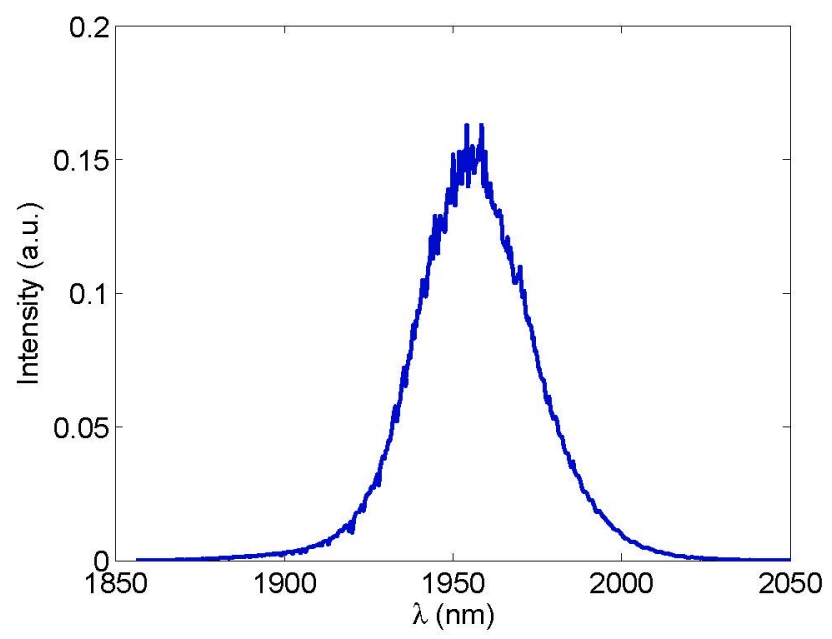

Figure 2. Spectrum of the Tm doped superfluorescent ASE fiber source

A time-domain fiber based OCT in the Michelson interferometer configuration using a $2 \mathrm{x} 2$ fiber coupler (Gooch \& Housego) is set up. The output of the ASE light is coupled into the input arm of the fibre coupler and split into two paths. One arm is used to illuminate a $50 \mathrm{~mm}$ focusing lens with a galvo scanning mirror which scans and collect light from the 
sample. The other light path illuminates a scanning reference mirror which changes the group delay and creates a coherent time gate in the mixed signal. The reference light and the sample light are recombined in the output arm of the fibre coupler and detected by an extended InGaAs detector sensitive up to $2.1 \mu \mathrm{m}$ in wavelength (Thorlabs).

The ASE source spectrum is shown in Fig. 2. The central wavelength of the source is $\sim 1950 \mathrm{~nm}$ and the FWHM bandwidth is $\sim 40 \mathrm{~nm}$ when operating at a power of $16 \mathrm{~mW}$. The power incident on the sample is $\sim 2 \mathrm{~mW}$. The theoretically expected FWHM axial resolution assuming a Gaussian spectrum is given by $\Delta z \sim 0.44 \lambda_{0}^{2} / \Delta \lambda$ which is $\sim 40 \mu \mathrm{m}$ in this case.

\section{RESULTS}

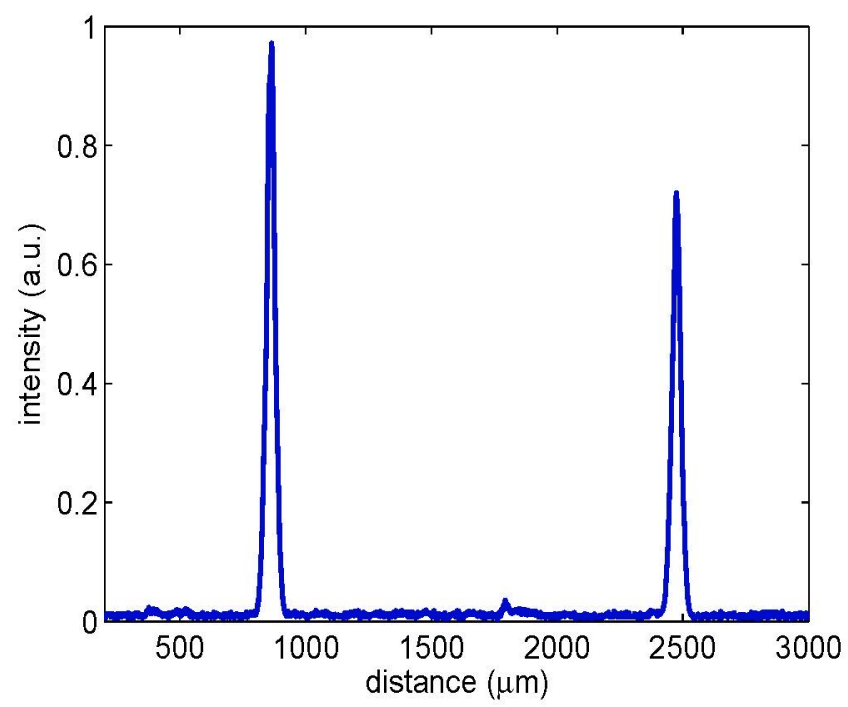

Figure 3. A depth profile (A-Scan) of a glass microscope slide using the $1950 \mathrm{~nm}$ OCT. The intensity scale is linear.

Figure 3 shows a depth profile obtained by the OCT of a glass microscope slide showing the axial resolution to be $\sim 35 \mu \mathrm{m}$. The measured optical thickness of the glass slide is $\sim 1.6 \mathrm{~mm}$ consistent with the known thickness.

Figure 4 shows a comparison of the cross-section images obtained with the 1950nm OCT and a 930nm OCT (Thorlabs OCP930SR) of a Titanium white oil paint on a glass microscope slide. The $930 \mathrm{~nm}$ OCT is a Fourier domain OCT with an axial resolution of $6.5 \mu \mathrm{m}$. The paint is highly transparent at $1950 \mathrm{~nm}$ and the thickness of the paint can be measured; however, at $930 \mathrm{~nm}$ the paint is highly scattering (dominated by multiple scattering) and opaque (the bottom of the glass slide cannot be seen at the expected position when the probe was moved closer to the sample).

Figure 5 shows a comparison of the $930 \mathrm{~nm}$ and $1950 \mathrm{~nm}$ OCT cross-section images for a layer of Prussian blue oil paint over a board prepared with a white gesso ground. The Prussian blue paint layer is strongly absorbing at $930 \mathrm{~nm}$ where only the air/paint interface is visible. However, at $1950 \mathrm{~nm}$ the paint layer can be clearly delineated showing some scattering from the paint layer and the paint/ground interface. Figure 5a shows the Prussian blue layer is $\sim 350 \mu \mathrm{m}$ in optical thickness or $\sim 230 \mu \mathrm{m}$ in physical thickness if we assume the paint refractive index to be $\sim 1.5$.

Figure 6 shows the image cube collected from a region where there is a charcoal underdrawing beneath the Prussian blue oil paint. Figure $6 c$ shows an average of slices extracted from the image cube around the depth of the underdrawing. The underdrawing is clearly visible in this averaged 'en face' slice. 


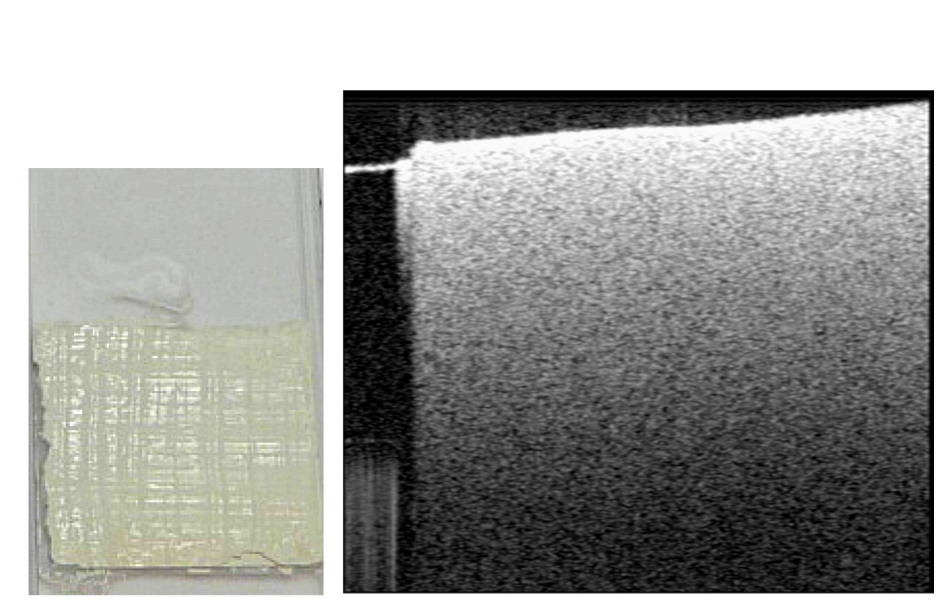

(a) (b)

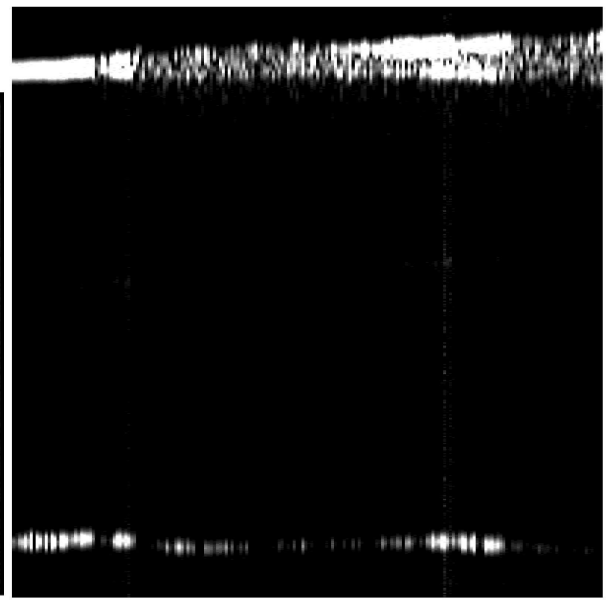

(c)

Figure 4. a) Titanium white oil paint on a glass microscope slide; b) 930nm OCT image showing multiple scattering from Ti White paint (image size: $3.3 \mathrm{~mm}$ wide by $1.6 \mathrm{~mm}$ deep) ; c) $1950 \mathrm{~nm}$ OCT image showing that the paint is transparent and even the bottom of the glass slide can be seen (image size: $3.3 \mathrm{~mm}$ wide by $1.9 \mathrm{~mm}$ deep) .

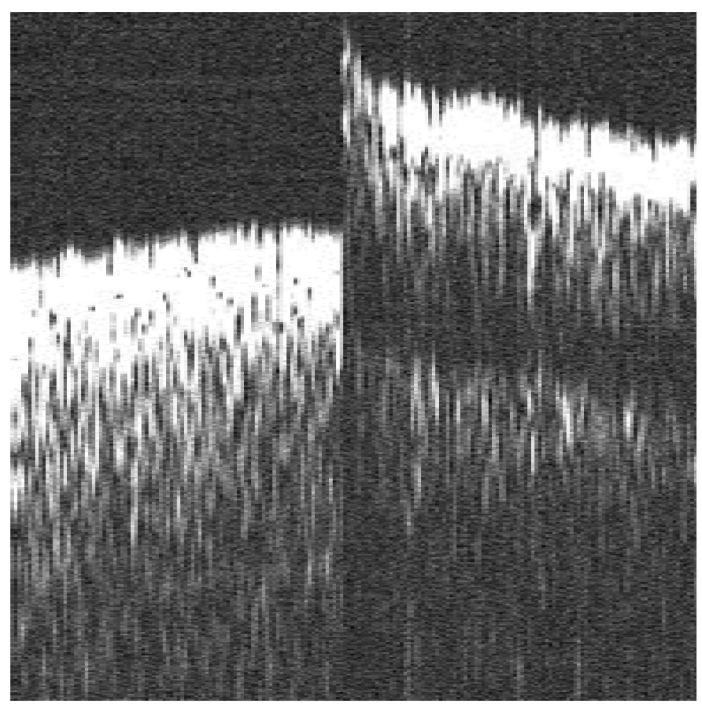

(a)

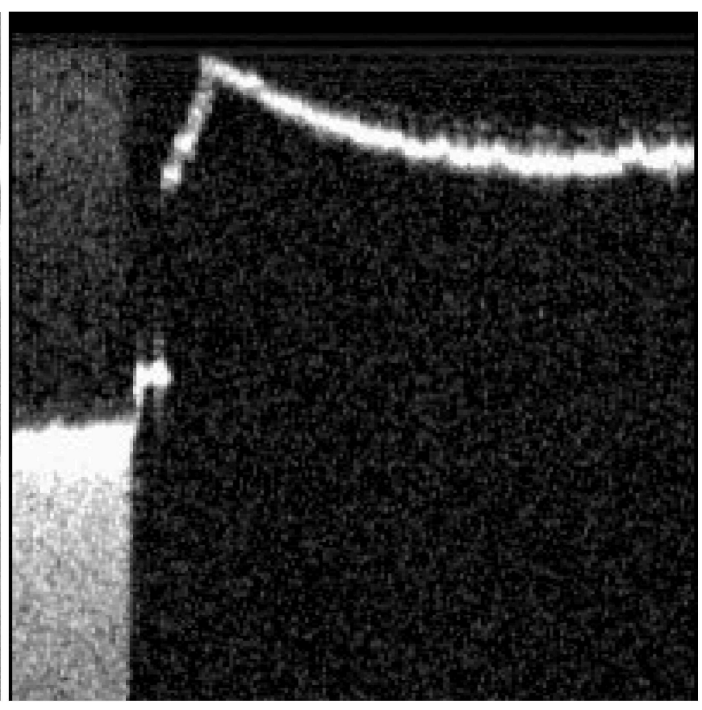

(b)

Figure 5. a) 1950nm OCT cross-section image of a layer of Prussian blue oil paint on a board covered with a white gesso ground (see Fig. 6a) ; b) 930 $\mathrm{nm}$ OCT cross-section image (B-scan); both images are $7 \mathrm{~mm}$ wide and $0.83 \mathrm{~mm}$ deep. 


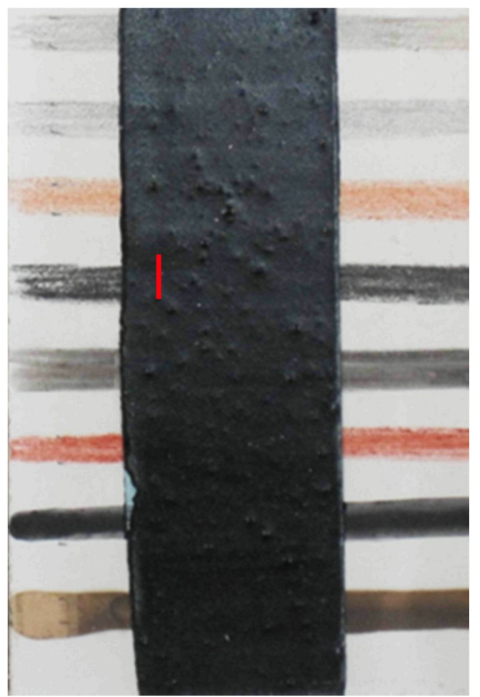

(a)

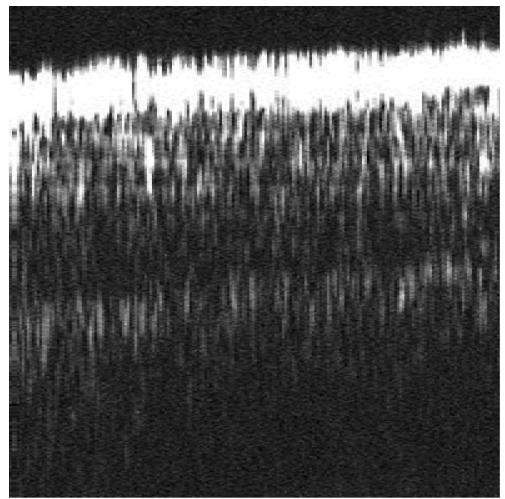

(b)

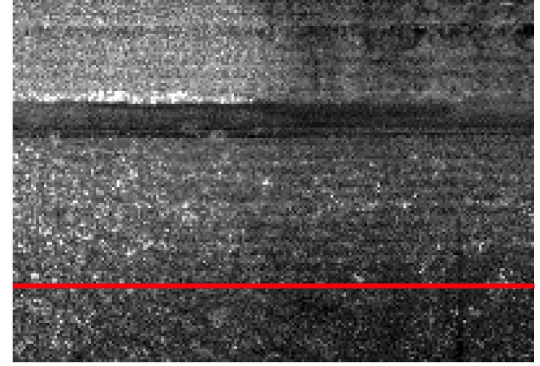

(c)

Figure 6. a) Color image of a Prussian blue paint layer above a substrate of white gesso ground painted with a number of different types of underdrawings; b) $1950 \mathrm{~nm}$ OCT cross-section at the red line segment in a) and c); b) an 'en face' slice averaged over the layers around the surface of the substrate; top part of the image is the region without paint and the lower part is the region covered by paint and the right half is painted with charcoal drawing.

\section{CONCLUSION}

Long wavelength OCT around $2 \mu \mathrm{m}$ has great potential in non-invasive imaging of paint layers since paint becomes more transparent at longer wavelengths. Future developments include using a broader band ASE source with a balanced detector and the development of a broad band swept source OCT.

\section{ACKNOWLEDGEMENTS}

Funding from UK AHRC and EPSRC Science \& Heritage Programme (Interdisciplinary Research Grant $\mathrm{AH} / \mathrm{H} 032665 / 1)$ is gratefully acknowledged. Paint samples are provided by the National Gallery. We are grateful to Gooch \& Housego plc. for providing the $2 \mu \mathrm{m}$ fiber couplers.

\section{REFERENCES}

[1] Targowski P., Iwanicka M., "Optical Coherence Tomography for structural examination of cultural heritage objects and monitoring of restoration processes - a review," Applied Physics A 106(2), 265-277 (2012).

[2] Liang H., Peric B., Hughes M., Podoleanu A., Spring M., Roehrs S., "Optical Coherence Tomography in Archaeology and Conservation Science - A new emerging field," Proc. SPIE 7139, 713915 (2008).

[3] Liang H., Cid M., Cucu R., Dobre G., Podoleanu A., Pedro J., Saunders D., "En-face optical coherence tomography - a novel application of non-invasive imaging to art conservation," Opt. Express 13, 6133-6144 (2005).

[4] Liang H., Lange R., Peric B., Spring M., "Optimum spectral window for imaging of art with optical coherence tomography," Applied Physics B doi 10.1007/s00340-013-5378-5, (2013).

[5] Shen D., Pearson L., Wang P., Sahu J., Clarkson W., "Broadband Tm-doped superfluorescent fiber source with $11 \mathrm{~W}$ single-ended output power," Opt. Express 16, 11021-11026 (2008). 